\title{
PMEA AND FIRST COUNTABLE, COUNTABLY PARACOMPACT SPACES
}

\author{
DENNIS K. BURKE
}

\begin{abstract}
The Product Measure Extension Axiom is used to prove a " $\theta$ expandable" type property for first countable, countably paracompact (countably metacompact) spaces. Among other results, it follows (under PMEA) that countably paracompact Moore spaces are metrizable, and first countable, countably paracompact Hausdorff spaces are strongly collectionwise normal with respect to compact sets.
\end{abstract}

1. Introduction. Under the assumption of the Product Measure Extension Axiom (PMEA) we show that countably paracompact Moore spaces are metrizable, and first countable, countably paracompact Hausdorff spaces are strongly collectionwise normal with respect to compact sets. These results follow from a theorem giving " $\theta$-expandable" type properties for first countable, countably paracompact or countably metacompact spaces.

All regular spaces are assumed to be $T_{1}$ but no other separation axioms are assumed unless otherwise stated. An ordinal number is the set of smaller ordinals, and $N=[1, \omega)$.

The Product Measure Extension Axiom (PMEA) says that for any set $A$ the usual product measure on $2^{A}$ can be extended to a $c$-additive measure $\mu$ defined on all subsets of $2^{A}$. That is, $\mu$ would be a measure defined for all subsets of $2^{A}$ such that

(i) $\mu(U)=2^{-k}$ whenever $U$ is a basic product neighborhood in $2^{A}$ restricted at $k$ coordinates;

(ii) whenever $\left\{B_{\alpha}: \alpha<\kappa\right\}$ is a disjoint collection of subsets of $2^{A}$ and $|\kappa|<c$ then $\mu\left(\bigcup_{\alpha<\kappa} B_{\alpha}\right)=\sum_{\alpha<\kappa} \mu\left(B_{\alpha}\right)$.

It is known that the consistency of PMEA would follow from the existence of a strongly compact cardinal and that the consistency of PMEA would imply the existence of a measurable cardinal. See $\left[\mathbf{F}_{\mathbf{2}}, \mathbf{N}\right.$ and $\left.\mathbf{T}\right]$ for a discussion of the status of PMEA as well as other applications of PMEA and weaker measure extension axioms. Our application of PMEA in Theorem 1.2 uses only the usual $\sigma$-additive property of a measure rather than the stronger additive property given in (ii) above.

The most notable application of PMEA in a topological setting is given in the provisional solution of the Normal Moore Space Problem by P. Nyikos in $[\mathbf{N}]$.

2. The main theorem. Our main result is Theorem 2.2. We need a simple lemma concerning the product measure on $2^{A}$.

Received by the editors December 28, 1983; presented to the Society, January 27, 1984.

1980 Mathematics Subject Classification. Primary 54D15, 54D18, 54E30.

Key words and phrases. Product Measure Extension Axiom, countably paracompact, countably metacompact, Moore space, collectionwise normal. 
2.1. LemmA. Suppose $A$ is a set, $\mu$ is the usual product measure on $2^{A}$ and $J_{1}, J_{2}, \ldots, J_{n}$ are finite disjoint subsets of $A$ with $\left|J_{i}\right|=k$ for every $i$. If $M_{i}$ denotes a basic neighborhood in $2^{A}$ with coordinates restricted at points in $J_{i}$ then $\mu\left(\bigcup_{i=1}^{n} M_{i}\right)>3 / 8$ for $n \geq 2^{k-1}$.

PROOF. For $n=2^{k-1}$ we observe there exists $\varepsilon_{r}>0$ for $3 \leq r \leq n$ such that

$$
\begin{aligned}
\mu\left(M_{1} \cup M_{2} \cup \cdots \cup\right. & \left.M_{n}\right) \\
= & \mu\left(M_{1}\right)+\mu\left(M_{1}-M_{2}\right)+\cdots+\mu\left(M_{n}-\left(M_{1} \cup \cdots \cup M_{n-1}\right)\right) \\
= & 2^{-k}+\left(2^{-k}-2^{-2 k}\right)+\left(2^{-k}-2 \cdot 2^{-2 k}+\varepsilon_{3}\right)+\cdots \\
& +\left(2^{-k}-(n-1) \cdot 2^{-2 k}+\varepsilon_{n}\right) .
\end{aligned}
$$

(For example, $\mu\left(M_{3}-\left(M_{1} \cup M_{2}\right)\right)=\mu\left(M_{3}\right)-\mu\left(M_{3} \cap M_{2}\right)-\mu\left(M_{3} \cap M_{1}\right)+\varepsilon_{3}$.) This gives $\mu\left(M_{1} \cup M_{2} \cup \cdots \cup M_{n}\right)>n \cdot 2^{-k}-(n-1) \cdot n \cdot 2^{-2 k-1}>3 / 8$.

The theorem below is proved for the first countable case. In a private communication to the author, P. Nyikos indicated he has extended the proof to the case when each point of $X$ has local character $<c$.

2.2. THEOREM. (PMEA) Suppose $X$ is a first countable, countably paracompact space (countably metacompact) space and $P=\left\{P_{\beta}: \beta<\lambda\right\}$ is a closed discrete collection in $X$. The canonical open cover $\left\{X-\bigcup_{\alpha \neq \beta} P_{\alpha}: \beta<\lambda\right\}$ has a sequence $\left\{\mathscr{H}_{n}\right\}_{n=1}^{\infty}$ of open refinements such that for any $x \in X$ there exists $m \in N$ where $H_{m}$ is locally finite (point finite) at $x$.

PROOF. We give the proof for the more difficult countably paracompact casethe countably metacompact case follows from a natural modification.

Let $\mu$ denote a ( $\sigma$-additive) measure defined for all subsets of $2^{\omega \times \lambda}$ and extending the usual product measure on $2^{\omega \times \lambda}$. For $x \in X$ let $\left\{V_{n}(x)\right\}_{1}^{\infty}$ be a decreasing open neighborhood base at $x$. We want to let an element of $2^{\omega \times \lambda}$ induce a natural countable partition of $\lambda$. For $f \in 2^{\omega \times \lambda}$ we let

$$
A_{\omega f}=\{\beta \in \lambda: f(\gamma, \beta)=0 \text { for all } \gamma<\omega\}
$$

and for $\alpha<\omega$

$$
A_{\alpha f}=\{\beta \in \lambda: f(\alpha, \beta)=1 \text { and } f(\gamma, \beta)=0 \text { for all } \gamma<\alpha\} .
$$

For $\alpha \leq \omega$ let

$$
W_{\alpha f}=X-\bigcup\left\{P_{\beta}: \beta<\lambda, \beta \notin A_{\alpha f}\right\} .
$$

Suppose $\mathcal{U}_{f}=\left\{U_{\alpha f}: \alpha \leq \omega\right\}$ is a precise locally finite open refinement of $\mathcal{W}_{f}=$ $\left\{W_{\alpha f}: \alpha \leq \omega\right\}$. For $p \in \bigcup\left\{P_{\beta}: \beta<\lambda\right\}$ and $k \in N$, let

$$
D(k, p)=\left\{f \in 2^{\omega \times \lambda}: V_{k}(p) \subset U_{\alpha f} \text { for some } \alpha \leq \omega\right\} .
$$

For every $n \in N$ and $p \in \bigcup\left\{P_{\beta}: \beta<\lambda\right\}$, there exists some $k(n, p) \in N$ such that

$$
\mu(D(k(n, p), p)) \geq 1-1 /\left(4 n \cdot 2^{n^{2}}\right)
$$

where we may assume $k(n, p)<k(n+1, p)$. Now, for $\beta<\lambda$, let

$$
H(n, \beta)=\bigcup\left\{V_{k(n, p)}(p): p \in P_{\beta}\right\}
$$

and $\mathscr{H}_{n}=\{H(n, \beta): \beta<\lambda\}$. It suffices to show that for any $x \in X$ there is $m \in N$ such that $H_{m}$ is locally finite at $x$. ( $\mathscr{H}_{m}$ may not cover $X$ but is certainly the open 
collection of interest since we may add $X-\bigcup P$ to $\mathscr{H}_{m}$.) To this end assume there exists some $x \in X$ such that $\operatorname{ord}\left(V_{n}(x), \mathscr{H}_{n}\right) \geq \omega$ for every $n \in N$. Pick distinct $\beta_{1}, \beta_{2}, \ldots$ from $\lambda$ such that $V_{i}(x) \cap H\left(i, \beta_{i}\right) \neq \emptyset$ for every $i \in N$ and choose $p_{i} \in P_{\beta_{i}}$ so that

$$
V_{i}(x) \cap V_{k\left(i, p_{i}\right)}\left(p_{i}\right) \neq \emptyset .
$$

Note that $V_{i}(x) \cap V_{r}\left(p_{i}\right) \neq \emptyset$ if $r \leq k\left(i, p_{i}\right)$. Let

$$
B_{n}=\left\{f \in 2^{\omega \times \lambda}: \operatorname{ord}\left(V_{n}(x), \mathcal{U}_{f}\right) \leq n\right\} ;
$$

then $B_{1} \subset B_{2} \subset \cdots$ and $\bigcup_{n \in N} B_{n}=2^{\omega \times \lambda}$ so $\mu\left(B_{m}\right)>7 / 8$ for some $m \in N$. For any $j \in N$ let $\gamma_{j i}=\beta_{j(m+1)+i}, 0 \leq i \leq m$, and $I_{j}=\left\{\gamma_{j i}: 0 \leq i \leq m\right\}$. Let $M_{j}$ be a basic neighborhood in $2^{\omega \times \lambda}$ defined by $M_{j}=\left\{f \in 2^{\omega \times \gamma}: f\left(r, \gamma_{j i}\right)=1\right.$ if and only if $r=i$ for $0 \leq r \leq m, 0 \leq i \leq m\}$. We see that for any $f \in M_{j}$ and $0 \leq i \leq m$ we have $\gamma_{j i} \in A_{r f}$ if and only if $r=i$. Also $\mu\left(M_{j}\right)=2^{-k}$ where $k=(m+1)^{2}$.

With the above description of $M_{j}$ and $k=(m+1)^{2}$, Lemma 2.1 says that $\mu\left(M_{1} \cup M_{2} \cup \cdots \cup M_{n}\right)>1 / 4$ if $n=2^{k-1}$. If $S=B_{m} \cap\left(M_{1} \cup M_{2} \cup \cdots \cup M_{n}\right)$ we have $\mu(S)>1 / 8$ so these exists some $j, 1 \leq j \leq n$, where

$$
\mu\left(B_{m} \cap M_{j}\right)>1 / 8 n=1 /\left(4 \cdot 2^{(m+1)^{2}}\right) .
$$

Consider

$$
E=\bigcap_{i=0}^{m} D\left(k\left(m+1, q_{j i}\right), q_{j i}\right)
$$

where $q_{j i}=p_{j(m+1)+i}$. By $(*)$ we have

$$
\mu(E) \geq 1-1 /\left(4 \cdot 2^{(m+1)^{2}}\right)
$$

so there exists some $g \in B_{m} \cap M_{j} \cap E$. Now notice:

(1) $g \in B_{m}$ implies ord $\left(V_{m}(x), \mathcal{U}_{g}\right) \leq m$.

(2) $g \in M_{j}$ implies $\gamma_{j i} \in A_{i g}, 0 \leq i \leq m$, so $q_{j i} \in U_{i g}$.

(3) $g \in E=\bigcap_{i=1}^{m} D\left(k\left(m+1, q_{j i}\right), q_{j i}\right)$ implies $V_{k\left(m+1, q_{j i}\right)}\left(q_{j i}\right) \subset U_{i g}$ for $0 \leq i \leq$ $m$ so $V_{m}(x) \cap U_{i g} \supset V_{j(m+1)+i}(x) \cap V_{k\left(j(m+1)+i, q_{j i}\right)}\left(q_{j i}\right) \neq \emptyset$.

Statement (3) says ord $\left(V_{m}(x), \mathcal{U}_{g}\right) \geq m+1$, contradicting statement (1). That completes the proof of the theorem.

The separation properties of first countable countably paracompact spaces are covered in the next section. We conclude this section with a couple of easy corollaries concerning the building of $G_{\delta}$-sets.

The proofs of Corollary 2.3 and the analogous Corollary 2.4 are left to the reader. We say $A$ is a regular $G_{\delta}$-set if $A$ can be expressed as $A=\bigcap_{n=1}^{\infty} \bar{U}_{n}$ where each $U_{n}$ is an open set containing $A$.

2.3. COROLLARY. (PMEA) In a first countable, countably metacompact space $X$ any closed set, which is the union of a discrete collection of $G_{\delta}$-sets, is a $G_{\delta}$-set. Hence, if $X$ is $T_{1}$, every closed discrete subset is a $G_{\delta}$-set.

2.4. COROLLARY. (PMEA) In a first countable countably paracompact space $X$ any set, which is the union of a discrete collection of regular $G_{\delta}-$ sets, is a regular $G_{\delta}$-set.

Certainly, countable metacompactness may always be considered as a weakening of the perfect condition (all closed sets are $G_{\delta}$-sets). In the first countable 
case, Corollary 2.3 suggests that a partial recovery of the perfect condition may be expected. This also suggests that many popular constructions, which yield first countable spaces with a closed discrete subset that is not a $G_{\delta}$-set, will not be countably metacompact, a fact not otherwise obvious in certain individual cases.

3. Separation properties. It is known $[\mathbf{M}]$ that if $F$ and $E$ are disjoint closed sets in a countably paracompact $X$ and $F$ is a regular $G_{\delta}$-set then $F$ and $E$ can be separated. The following Theorem 3.1(a) can be viewed as an extension of this result. Part (b) gives the desired collectionwise separation.

THEOREM. (PMEA) Suppose $X$ is a first countable, countably paracompact space, $\left\{F_{\alpha}: \alpha<\lambda\right\}$ is a closed discrete collection and $\left\{U_{n \alpha}: n \in N, \alpha<\lambda\right\}$ is an open collection in $X$ such that

$$
F_{\alpha} \subset \bigcap_{n=1}^{\infty} U_{n \alpha} \subset \bigcap_{n=1}^{\infty} \bar{U}_{n \alpha}=D_{\alpha} \quad \text { for each } \alpha<\lambda .
$$

(a) If $W$ is an open set such that $\bigcup_{\alpha<\lambda} D_{\alpha} \subset W$ then there exists an open set $U$ such that $\bigcup_{\alpha<\lambda} F_{\alpha} \subset U \subset \bar{U} \subset W$.

(b) If $D_{\alpha} \cap F_{\beta}=\emptyset$ for all $\alpha, \beta \in \lambda, \alpha \neq \beta$, then $\left\{F_{\alpha}: \alpha<\lambda\right\}$ can be separated by a collection of open sets.

PROOF. It follows from Theorem 2.2 that there exists a sequence $\left\{\mathscr{H}_{n}\right\}_{n=1}^{\infty}$ of open collections where

$$
\mathscr{H}_{n}=\left\{H_{n \alpha}: \alpha<\lambda\right\}, \quad F_{\alpha} \subset H_{n \alpha} \subset U_{n \alpha}, \quad H_{(n+1) \alpha} \subset H_{n \alpha}
$$

and for any $x \in X$ there is some $m \in N$ where $\mathscr{H}_{m}$ is locally finite at $x$.

For the proof of (a) let $V_{n}=X-\operatorname{cl}\left(\bigcup_{\alpha<\lambda} H_{n \alpha}\right)$. The open collection $\{W\} \cup$ $\left\{V_{n}: n \in N\right\}$ covers $X$ so there must be a precise locally finite open refinement which we may assume is of the form $\{W\} \cup\left\{G_{n}: n \in N\right\}$ where $G_{n} \subset V_{n}$. If $U=X-\operatorname{cl}\left(\bigcup_{n=1}^{\infty} G_{n}\right)$ it follows that $\bigcup_{\alpha<\lambda} F_{\alpha} \subset U \subset \bar{U} \subset W$.

For the proof of (b) define

$$
K_{n \alpha}=H_{n \alpha}-\operatorname{cl}\left(\bigcup\left\{H_{n \beta}: \beta<\lambda, \beta \neq \alpha\right\}\right)
$$

for each $\alpha<\lambda, n \in N$, and let $K_{\alpha}=\bigcup_{n=1}^{\infty} K_{\alpha n}$. If follows that $F_{\alpha} \subset K_{\alpha}$ and $K_{\alpha} \cap K_{\beta}=\emptyset$ if $\alpha \neq \beta$, so $\left\{K_{\alpha}: \alpha<\lambda\right\}$ is the desired separating open collection.

Both parts of the above theorem can be used in certain cases to obtain "strong collectionwise" separation; that is, separation by discrete collections of open sets. The corollary below also answers a question asked by S. Watson [W] of whether PMEA implies first countable, countably paracompact spaces are collectionwise Hausdorff.

3.2. COROLlary. (PMEA) (a) A first countable, countably paracompact $T_{2}$ space $X$ is strongly collectionwise normal with respect to compact sets.

(b) A first countable, countably paracompact space $X$ is strongly collectionwise normal with respect to regular $G_{\delta}$-sets.

Proof. For (a) assume $\left\{F_{\alpha}: \alpha<\lambda\right\}$ is a discrete collection of compact sets where $F_{\alpha} \neq F_{\beta}$ if $\alpha \neq \beta$. Since a first countable, countably paracompact $T_{2}$ space is regular $[\mathbf{A}]$ we can find open sets $U_{\alpha}, \alpha<\lambda$, such that $F_{\alpha} \subset U_{\alpha}$ and 
$\bar{U}_{\alpha} \cap F_{\beta}=\emptyset$ if $\alpha \neq \beta$. By 3.1(b) we see there exist open sets $W_{\alpha}, \alpha<\lambda$, such that $F_{\alpha} \subset W_{\alpha}$ and $W_{\alpha} \cap W_{\beta}=\emptyset$ if $\alpha \neq \beta$. Now find open sets $V_{\alpha}, \alpha<\lambda$, such that $F_{\alpha} \subset V_{\alpha} \subset \bar{V}_{\alpha} \subset W_{\alpha}$. An application of 3.1(a) gives an open set $H$ such that

$$
\bigcup_{\alpha<\lambda} F_{\alpha} \subset H \subset \bar{H} \subset \bigcup_{\alpha<\lambda} W_{\alpha} .
$$

The collection $\left\{H \cap W_{\alpha}: \alpha<\lambda\right\}$ is the desired discrete separating open family.

For (b) assume $\left\{F_{\alpha}: \alpha<\lambda\right\}$ is a discrete collection of regular $G_{\delta}$-sets. A direct application of 3.1(b) gives an open separating family $\left\{W_{\alpha}: \alpha<\lambda\right\}$, and 3.1(a) gives an open set $H$ such that $\bigcup_{\alpha<\lambda} F_{\alpha} \subset H \subset \bar{H} \subset \bigcup_{\alpha<\lambda} W_{\alpha}$. The collection $\left\{H \cap W_{\alpha}: \alpha<\lambda\right\}$ is a discrete separating open family.

4. Countably paracompact Moore spaces. The main purpose of this section is to show that countably paracompact Moore spaces are metrizable under PMEA. We actually prove a stronger result in Theorem 4.2 . It will be convenient to have the following standard lemma.

4.1. LEMMA. A space $X$ is normal if and only if whenever $F$ and $E$ are disjoint closed sets in $X$ there exist open sets $H_{n}$, for $n \in N$, such that $F \subset \bigcup_{n=1}^{\infty} H_{n}$ and each $\bar{H}_{n} \cap E=\emptyset$.

4.2. THEOREM. (PMEA) A first countable, countably paracompact, subparacompact $T_{2}$ space $X$ is paracompact.

Proof. It suffices to show $X$ is normal since (under PMEA) this gives collectionwise normality $[\mathbf{N}]$ and collectionwise normal subparacompact spaces are paracompact. Let $F$ and $E$ be disjoint closed sets in $X$. Since $X$ is actually regular there is a collection $U=\left\{U_{\alpha}: \alpha<\lambda\right\}$ of open subsets of $X$ such that $F \subset \bigcup_{\alpha<\lambda} U_{\alpha}$ and $\bar{U}_{\alpha} \cap E=\emptyset$, all $\alpha<\lambda$. Using the subparacompactness of $X$ there is a $\sigma$-discrete closed collection $\mathcal{F}$ of subsets of $F$, covering $F$, of the form

$$
\mathcal{F}=\left\{F_{n \alpha}: n \in N, \alpha<\lambda\right\}
$$

where $F_{n \alpha} \subset U_{\alpha}$ and each $\mathcal{F}_{n}=\left\{F_{n \alpha}: \alpha<\lambda\right\}$ is discrete (some $F_{n \alpha}$ may be empty). By 3.1(a) we see there is an open set $H_{n}$, for each $n \in N$, such that $\bigcup_{\alpha<\lambda} F_{n \alpha} \subset H_{n} \subset \bar{H}_{n} \subset X-E$. Apply Lemma 4.1 and the proof is complete.

We remark that standard techniques (with more cumbersome notation) would extend the proof of 4.2 to "submetacompact" in place of "subparacompact". P. Nyikos has also noticed this using a different approach.

The proof of the next corollary is now immediate. Recall that W. Fleissner has shown $\left[\mathbf{F}_{\mathbf{2}}\right]$ that separable countably paracompact Moore spaces are metrizable under $(\mathrm{CH})$.

4.3. COROLLARY. (PMEA) Every countably paracompact Moore space is metrizable.

\section{REFERENCES}

[A] C. E. Aull, A note on countably paracompact spaces and metrization, Proc. Amer. Math. Soc. 16 (1965), 1316-1317.

[F $\left.\mathbf{F}_{1}\right]$ W. G. Fleissner, Separation properties in Moore spaces, Fund. Math. 98 (1978), 279 286. 
$\left[\mathbf{F}_{2}\right] \ldots$, The normal Moore space conjecture and large cardinals, Handbook of Set-Theoretic Topology (to appear).

[M] J. Mack, Countable paracompactness and weak normal properties, Trans. Amer. Math. Soc. 148 (1970), 265-272.

[N] P. J. Nyikos, A provisional solution to the normal Moore space problem, Proc. Amer. Math. Soc. 78 (1980).

[T] F. D. Tall, Normality versus collectionwrise normality, Handbook of Set-Theoretic Topology (to appear).

[W] W. S. Watson, Applications of set theory to general topology, Thesis, University of Toronto, 1982.

Department of Mathematics, Miami University, OXford, Ohio 45056 